\title{
EDAD CRONOLÓGICA EN LA RESOLUCIÓN ESPONTÁNEA DEL REFLUJO VESICO URETERAL
}

\author{
P. Baquedano', A. Nardiello², P. Orellana3, M. Díaz', y E. Lagomarsino².
}

'Uróloga Infantil. Departamento de Urología. Pontificia Universidad Católica de Chile. Santiago de Chile. ${ }^{2}$ Nefróloga Infantil. Departamento de Pediatría. Pontificia Universidad Católica de Chile. Santiago de Chile. 3Médico Nuclear. Departamento de Radiología. Pontificia Universidad Católica de Chile. Santiago de Chile.

\begin{abstract}
Resumen.- OBJETIVO: El Reflujo Vésico Ureteral (RVU) puede tener un tratamiento médico, eso implica administración de antibióticos profilácticos, control urocultivo seriado y exámenes de imágenes, como Uretrocistografía (UCG) o Cistografía Isotópica Directa (CID) anualmente; o bien un un tratamiento quirúrgico, cuando falla el tratamiento médico, ya sea por ITU recurrentes o daño renal. Analizar a qué edad cronológica del paciente, se resuelven espontáneamente los RVU, de forma tal de saber a que edad debemos controlar con UCG más precisamente, evitando el trauma del exámen y la irradiación, pero también evitando dar antibioticos profilacticos sin necesidad real. Asociar la edad de resolución con el grado y lateralidad del RVU y con el género del paciente.
\end{abstract}

MÉTODOS: Estudio retrospectivo de pacientes controlados en el Hospital Clínico de la Universidad Católica de Chile, entre los años 1996 y 2004. Criterios de inclusión fueron: pacientes con diagnóstico de RVU I, II, y III realizado por UCG, y UCG o CID de control con ausencia de RVU en forma espontánea, sin cirugía urológica, sin disfunción vesical u otras alteraciones del tracto urinario. Con estos criterios se incluyeron 156 pacientes.

RESULTADOS: $73.5 \%$ son de sexo femenino, la edad promedio al momento de la resolución fue de 52,7 \pm 36 meses. Un 74,3\% de los pacientes tenía RVU unilateral. Las edades de resolución entre los RVU bilaterales y unilaterales, y sus distintos grados, no presentaron diferencias estadísticamente significativas. En la resolución por sexo, existe una diferencia estadísticamente significativa $(p<0,0001)$ entre hombres y mujeres (los primeros resuelven 12 a 17 meses antes).

CONCLUSIONES: Los hombres mejoran antes el RVU que las mujeres y no encontramos diferencia significativa entre bilateral y unilateral, ni por grado de RVU, en la edad de resolución de los RVU I, II y III. La UCG o CID para controlar RVU deberíamos pedirla en relación a la edad cronológica más probable de resolución, en general alrededor de los 4 años de edad, y no 1 vez al año, evitando así la irradiación y el trauma, considerando especialmente el sexo del niño.

Palabras clave: Reflujo vesico ureteral. Resolución espontánea. Infección tracto urinario. Uretrocistografía miccional seriada. 
Summary.- OBJECTIVES: Vesicoureteral reflux (VUR) may have medical management, which implies prophylactic antibiotic therapy, control with serial urine cultures and imaging tests, such as annual voiding cystourethrogram (VCUG) or direct isotopic cystogram (DIC); or it may be surgical, when medical treatment fails, due to recurrent UTI or renal damage. We analyze the chronological age of the patient at which VUR resolve spontaneously, so that we know when we should have a more precise control with VCUG, avoiding a traumatic test and irradiation, but also avoiding to give prophylactic antibiotic without real need. We aim to associate age of resolution with VUR grade and side, and patient's gender.

METHODS: Retrospective study of patients with VUR under control at the Hospital Clínico of the Catholic University of Chile between 1996 and 2004. Inclusion criteria: patients with the diagnosis of VUR grades I, II, and III obtained by VCUG, and spontaneous resolution of VUR on control VCUG or DIC, with no urological surgery, without bladder dysfunction or other urinary tract disorders. 156 patients were included.

RESULTS: $73.5 \%$ are females, with a mean age at the time of resolution of $52.7+-36$ months. $74.3 \%$ of the patients had unilateral VUR. There were not statistically significant differences in the age of resolution between unilateral or bilateral VUR, or between different grades. There is a statistically significant difference in the age of resolution comparing gender $(p<0.0001)$, resolving between 12 and 17 months before in males.

CONCLUSIONS: VUR improves earlier in males than females, without differences between unilateral-bilateral, or VUR grade I, II and III. The control VCUG or DIC should be indicated in relation to the most probable chronological age of resolution, generally around 4 years of age, and not once a year, so avoiding irradiation and trauma, with a special consideration to the child gender.

Keywords: Vesicoureteral reflux. Spontaneous resolution. Urinary tract infection. Voiding cystourethrogram.

\section{INTRODUCCIÓN}

El Reflujo Vesico Ureteral (RVU) es una patología de la vía urinaria, que determina una terapia adecuada para proteger el parénquima renal (1). Por décadas el tratamiento fue de resorte quirúrgico $(2,3)$, hasta que Hutch en 1961, planteó la teoría de maduración del RVU, como resultado de la elongación del trayecto submucoso del uréter, y por lo tanto la posibilidad de resolución espontánea $(4,5)$.

Esto abrió la posibilidad de un tratamiento distinto del RVU, y desde esa época se ha ido progresando hacia un tratamiento cada vez más conservador, siempre que se preserve la función renal. Esto avalado por diversos estudios como el de Birmingham Reflux Study Group y el International Reflux Study Comittee en que concluyen que el daño renal es independiente del tipo de tratamiento $(1,6,7)$.

El tratamiento médico requiere la estrecha colaboración de la familia, la mantención de la profilaxis antibiótica, el control de urocultivos seriados y la realización de Uretrocistografía (UCG) o Cistografía Isotópica Directa (CID) seriada, ambos procedimientos invasivos e incómodos para los pacientes y sus padres (8). La recomendación por mucho tiempo fue de realizar la UCG en forma anual, para corroborar la presencia o no de RVU, sin embargo cada vez más en el último tiempo se ha tendido ha realizar estudios que muestren el beneficio de espaciar aún más los controles de UCG a cada 2 años en RVU moderados y a cada 3 años en RVU severos (9). Todo esto teniendo en consideración la carga económica para los padres y efectos colaterales para los pacientes (8). Sin embargo con este tipo de planteamiento se corre el riesgo de prolongar inútilmente la mantención de profilaxis antibiótica $(9,10)$.

Por este motivo el objetivo de nuestro trabajo es poder determinar a que edad cronológica del niño se resuelve más frecuentemente el RVU, y su relación con el grado, género del paciente y lateralidad, de forma tal de no prolongar innecesariamente la profilaxis antibiótica, y tampoco someter anualmente a los niños a radiación y trauma por la UCG, y realizar éste examen lo más cercano a la edad de resolución para cada caso.

\section{MATERIAL Y MÉTODO}

Se realizó un estudio retrospectivo de pacientes controlados en los policlínicos de nefrología y urología de la Red de Salud de la Universidad Católica de Chile, entre los años 1994 y 2004 . Se obtuvo los datos de las fichas clínicas de 156 pacientes con diagnóstico de RVU de resolución espontánea. Se incluyeron en el estudio todos los pacientes con diagnóstico de RVU grado I, II o III, obtenido a través de UCG y el antecedente de resolución espontánea documentado por una nueva UCG o CID. Se excluyeron los pacientes con RVU, con grados IV y $\mathrm{V}$ por ser un número muy reducido de pacientes ( 5 en total, 
TABLA I. RESULTADOS SEGÚN EDAD CRONOLÓGICA.

\begin{tabular}{|c|c|c|c|c|c|c|c|c|}
\hline $\begin{array}{c}\text { Edad en } \\
\text { años }\end{array}$ & $\begin{array}{c}\% \\
\text { Total }\end{array}$ & Grado I & Grado II & Grado III & $\begin{array}{c}\% \\
\text { Mujeres }\end{array}$ & $\begin{array}{c}\% \\
\text { Hombres }\end{array}$ & $\begin{array}{c}\% \\
\text { Unilateral }\end{array}$ & $\begin{array}{c}\% \\
\text { Bilaterales }\end{array}$ \\
\hline 1 & 4 & 10 & & 10 & 4 & 6 & 10 & 4 \\
\hline 2 & 21 & 19 & 16 & 20 & 12 & 38 & 19 & 18,5 \\
\hline 3 & 28 & 15 & 31 & 32 & 28 & 28 & 32 & 27 \\
\hline 4 & 18 & 25 & 23 & 9 & 18 & 18 & 15 & 20 \\
\hline 5 & 14 & 25 & 13 & 9 & 18 & 6 & 7 & 15 \\
\hline 6 & 9 & 6 & 10 & 9 & 12 & 4 & 12 & 8,5 \\
\hline 7 & 6 & & 4 & 11 & 8 & & 4 & 6 \\
\hline
\end{tabular}

no representativo para someterlo a análisis estadísti$\mathrm{co})$, aquellos con antecedentes de disfunción vesical, entendiendo por esta la presencia de incontinencia, urgencia o frecuencia miccional alterada, cirugía urológica u otra patología de la vía urinaria distinta al RVU, y todos los pacientes con resolución quirúrgica de su RVU. De cada ficha se consignó el género del paciente, el grado de RVU según la clasificación Internacional (1 1), la lateralidad y la edad a la que se obtuvo desaparición del RVU en la UCG.

Todos los pacientes durante su seguimiento se mantuvieron en profilaxis antibiótica y se les realizó UCG anualmente. No todos los pacientes fueron diagnosticados en la Red de Salud de nuestro Hospital, pero todos al momento de la resolución eran controlados por nosotros.

Evolución Grupo Total

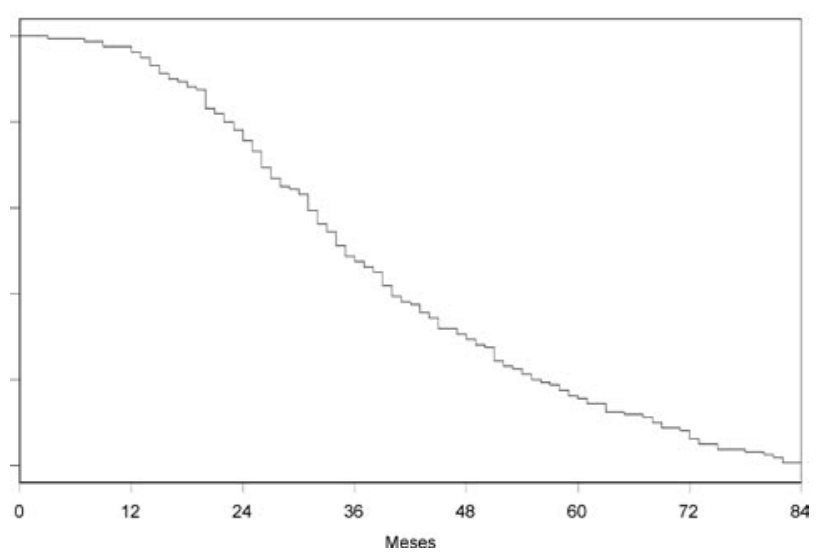

FIGURA 1. Resolución espontánea del total del grupo analizado.
Los datos obtenidos fueron analizados con curvas de Kaplan Meier, para estimar la tasa de resolución del RVU según edad, género, lateralidad y grado del RVU.

\section{RESULTADOS}

De los 156 pacientes un 73,5\% eran de género femenino, y un $74,3 \%$ de los RVU eran unilaterales.

Al analizar según el grado encontramos que al momento del diagnóstico el $26,5 \%$ eran grado I, el $40,5 \%$ eran grado II y el $33 \%$ grado III (Tabla I).

No hubo diferencias por lateralidad, ni por grado de RVU, en hombres y mujeres, manteniendo

Evolución por Lateralidad

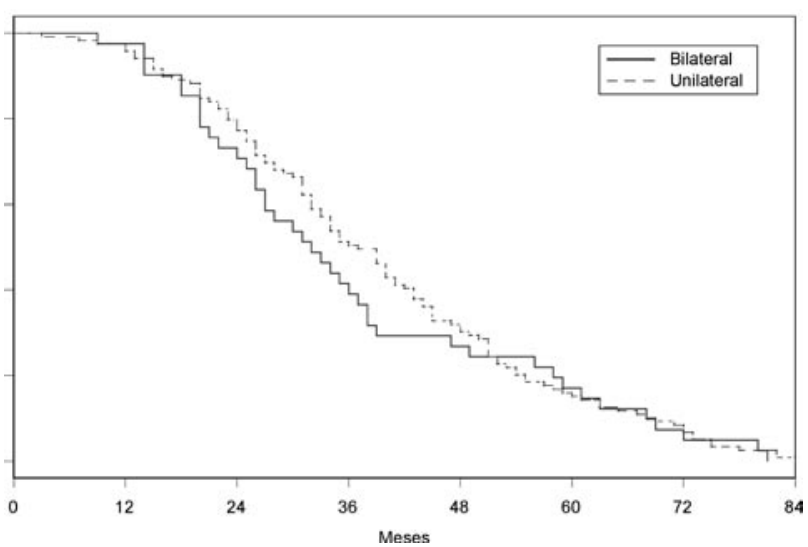

FIGURA 2. Resolución espontánea según lateralidad. 
Evolución Grupo con Grado 1

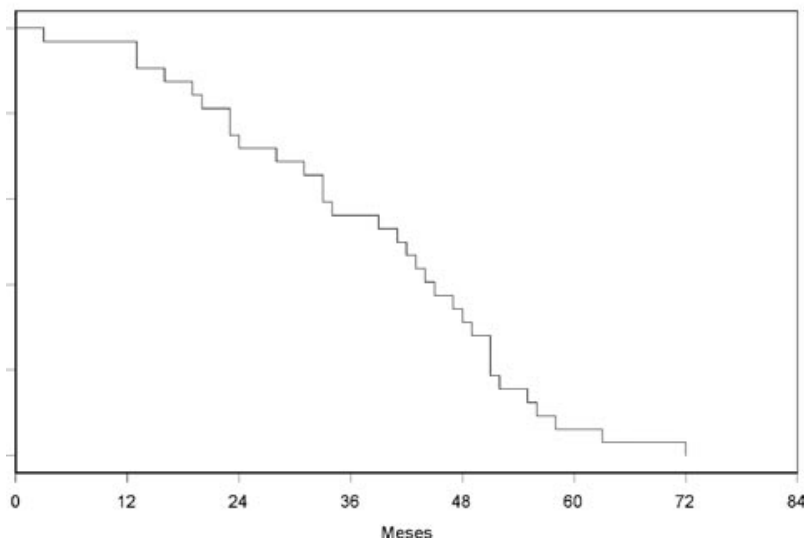

Evolución Grupo con Grado 2

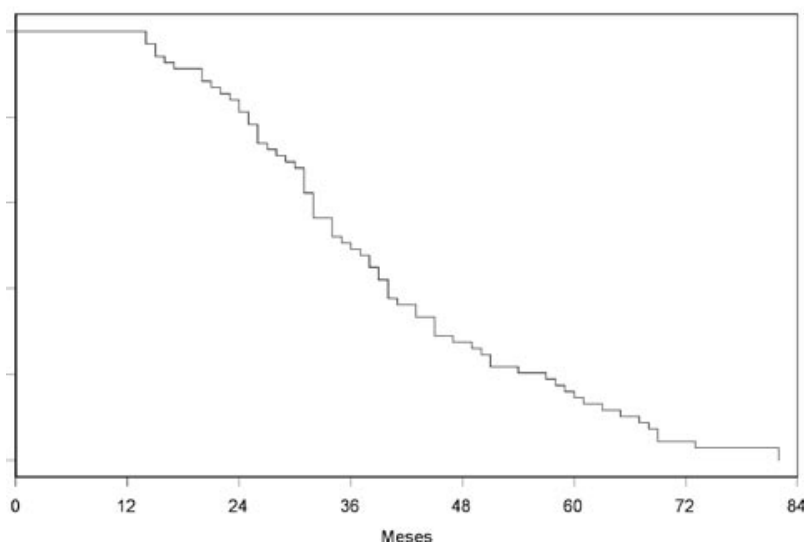

Evolución Grupo con Grado 3

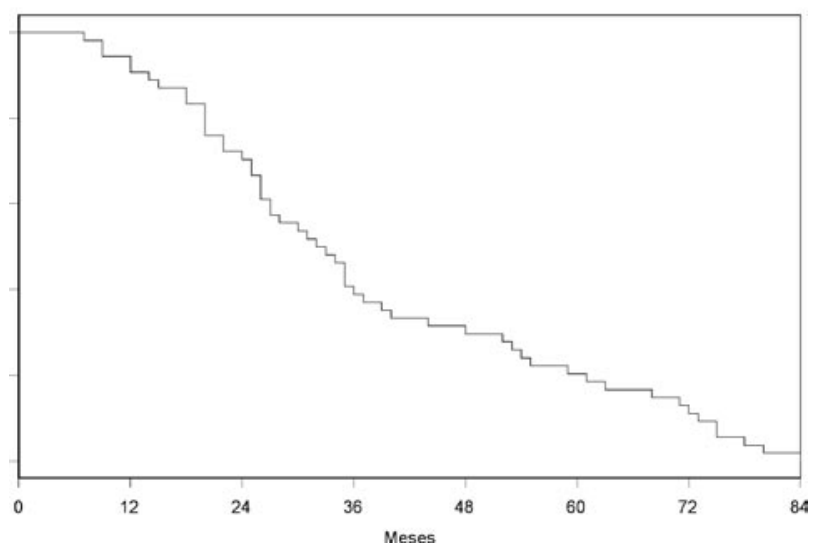

FIGURA 3. Resolución espontánea según grado de RVU. porcentajes similares al grupo total, es decir en relación mujer:hombre de 3:1.

El promedio de edad al momento de resolución del RVU, del grupo general, fue de 52,7 \pm 36 meses (Figura 1), al analizar la edad de resolución entre los RVU unilaterales y los bilaterales, no encontramos diferencia estadísticamente significativa, siguiendo ambos grupos aproximadamente la misma tendencia (Figura 2). Igual resultado se obtuvo al analizar la edad de resolución según el grado de RVU, alrededor de un $30 \%$ de los pacientes con grado II y III presentaban resolución espontánea alrededor de los 3 años, en cambio para el grado I un $50 \%$ de los pacientes se resolvía incluso a los 4 y 5 años, sin embargo estos resultados no tienen diferencia estadísticamente significativa (Figura 3).

Al analizar los resultados por género, observamos que más del $40 \%$ de los pacientes hombres presenta mejoría del RVU a los 2 años de vida, en relación a las mujeres que en su mayoría la presenta luego de los 3 años ( $p<0,005)$ (Figura 4).

\section{DISCUSIÓN}

Desde hace muchas décadas, el tratamiento conservador del RVU se ha sustentado en la observación de la regresión espontánea del RVU primario, regresión que aumenta con la edad de los pacientes $(3,5,12)$.

Diversos estudios sobre RVU, muestran una incidencia mayor en mujeres en relación a los hombres, en general muy cercana al $3: 1(13,14)$, al igual que el

\section{Evolución por Sexo}

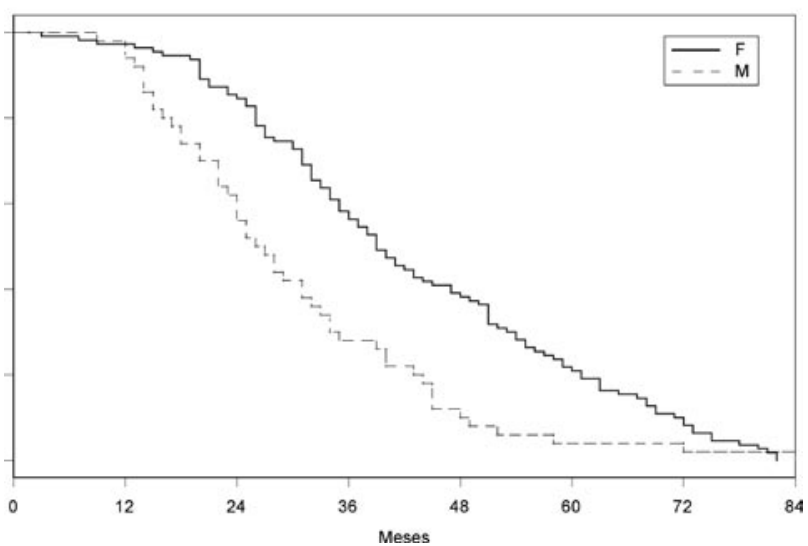

FIGURA 4. Comparación por género. 
encontrado en nuestro grupo, así como la distribución según los grados de RVU, siendo el grado II el que presenta mayor porcentaje, seguidos del grado I y III en porcentajes similares (14), sin llegar a presentar una diferencia estadísticamente significativa.

El promedio de edad en la que se produjo la resolución espontánea de los pacientes fue de 52,7 meses +36 meses, es decir alrededor de los 4 años y medio. Si esto lo analizamos por lateralidad, no encontramos diferencias entre ambos grupos, resolviendo en promedio a la edad del grupo total, independiente de ser unilaterales o bilaterales (Figura 2), a diferencia de lo encontrado por algunos autores como Schwab y cols. que describe que los RVU I al III unilaterales resuelven más rápidamente que sus semejantes bilaterales, sin llegar a tener diferencia estadísticamente significativa. Sin embargo resultados similares al nuestro han sido reportados por otros autores como Kuczynsca R y cols. (15) y Arant y cols. (16).

El análisis de cuál es la edad de resolución espontánea de los RVU según su grado, no mostró diferencias significativas entre los tres grupos (Figura 3), es decir independiente del grado de RVU que tenga un paciente, la edad más probable en la que se resolverá espontáneamente no tiene que ver con este parámetro. Estos resultados podrían interpretarse como que difieren de los encontrados en otros trabajos en que se analizan la resolución por grado de RVU, sin embargo hay que recordar que estos trabajos analizan la probabilidad de resolución espontánea, y comparan pacientes con RVU resuelto espontáneamente de los que se resuelven por otra vía. En este sentido se ha encontrado que tiene mayor posibilidad de resolver en forma espontánea los RVU leves (I, II) en relación a los moderados (III, IV) $(3,4,6,12,14,17)$.

Nuestro trabajo lo que concluye es que un paciente con RVU grado I no va a resolver necesariamente, a menor edad que otro, con iguales características y con un RVU grado III. El otro aspecto importante a señalar, es que nuestro estudio analiza sólo los RVU I al III, no incluyendo los de mayor severidad y menor posibilidad de resolución espontánea, que varía según lo descrito por distintos autores entre un $9 \%$ a un $15 \%$ anual $(18,19)$. Sin embargo estudios como el de Schwab, que compara los RVU II y III, encuentra una tasa de resolución similar, incluso autores como Mc Lorie (20) al comparar RVU grado III a V no encontraron diferencia estadísticamente significativa en su tasa de resolución.

En relación a la edad de resolución según el género del paciente, encontramos diferencia esta- dísticamente significativa $(p<0,005)$ entre hombres y mujeres (Figura 4), por la cual los hombres, independiente de las otras variables se mejoran a más temprana edad (entre los 24 y 36 meses, antes que la edad promedio del grupo general). Este punto ha sido descrito de forma similar por otros autores, que describen que los varones tienden a mejorarse más rápidamente del RVU que las mujeres $(12,21,22)$.

Esta última variable por lo tanto, debería ser fundamental al momento de tomar la decisión de cuándo realizar la UCG de control, en relación a la edad en que resuelve la mayoría de los pacientes, y por ejemplo un paciente varón en que el diagnóstico de RVU se realiza a los 2 años, tal vez sea recomendable controlarle en 1 año la próxima UCG, pero si es una mujer debería posponerse hasta que esté más cercana a los 4 años.

\section{CONCLUSIONES}

Siempre con el objetivo de preservar la función renal en los pacientes con RVU, y someterlos al menor daño posible, ya sea por exceso de exámenes (UCG) o exceso de terapia (antibióticos profilacticos), sería recomendable fijar la periodicidad con que se controlará la UCG, según la edad de cada paciente y el género, y no según el tiempo de evolución del RVU. Recordando que la edad en que resuelve espontáneamente la mayoría de los pacientes es alrededor de los 4 años, y considerando que los varones tienen en general mayor posibilidad de mejorar entre los 2 a 3 años de edad. Otro aspecto importante es que no tiene mayor significancia para el momento de resolución, el presentar RVU de mayor grado (entre I, II y III), así como tampoco el ser bilateral.

Estos resultados pretenden orientar a la realización racional de UCG en niños con RVU, con la finalidad de realizar el menor número de exámenes posibles, sin poner en riesgo al niño.

\section{BIBLIOGRAFÍA y LECTURAS RECOMENDADAS ( ${ }^{*}$ lectura de interés $y^{* *}$ lectura fundamental)}

1. ARANT, B.S.: "Vesicoureteric reflux and renal injury". Am. J. Kidney. Dis., 17: 491, 1991.

2. HUTCH, J.A.: "Theory of maturation of the intravesical ureter". J. Urol., 86: 534, 1961.

3. EDWARDS, D.; NORMAND, I.C.S.; PRESCOD, N. y cols.: "Dissapearance of vesicoureteric reflux during long term prophylaxis of urinary tract infection in children”. BMJ, 2: 285, 1977. 
4. ROLLESTON, G.L.; SHANNON, F.T.; UTLEY, W.L.F.: "Relationship of infantile vesicopureteric reflux to renal damage". BMJ, 1: 460, 1970.

5. ALADJEM, M.; BOICHIS, H.; HERTZ, M. y cols.: "The conservative management of vesicoureteric reflux: A review of 121 children". Pediatrics, 65: 78, 1980.

6. LEVITT, S.B.; DUCKETT, J.; SPITZER, A. y cols.: "Medical versus surgical treatment of primary vesicoureteral reflux. Report of international reflux study committee". Pediatrics, 67: 392, 1981.

7. BIRMINGHAM REFLUX STUDY GROUP.: "Prospective trial of operative versus non operative treatment of severe vesicoureteric reflux in children: Five years observation”. BMJ, 295: 237, 1987.

8. PHILIPS, D.; WATSON, A.R.; McKINLEY, D.: "Distress and micturating cystourethrography: Does preparation help?". Acta Pediatr., 87: 175, 1998.

9. THOMPSON, M.; SIMON, S.; SHARMA, V. y cols.: "Timing of follow up voiding cystourethrogram in children with primary vesicoureteral reflux: Development and application of clinical algorithm". Pediatrics, 115: 426, 2005.

10. GOSSENS, H.; SPRENGER, M.J.W.: "Community acquired infections and bacterial resistance". BMJ, 317: 654, 1998.

11. LEBOWITZ, R.L.; OLBING, H.; PARKKULAINEN, K.V. y cols.: "International reflux study in children writing committee: International system of radiographic grading of vesicoureteral reflux". Pediatr. Radiol., 15: 105, 1985.

12. SCHWAB, C.W.; WU, H.Y.; SELMAN, H. y cols.: "Spontaneous resolution of vesicoureteral reflux: A 15 year perspective". J. Urol., 168: 2594, 2002.

13. SMELLIE, J.M.; JODAL, U.; LAX, H. y cols.: "Outcome at 10 years of severe vesicoureteric reflux managed medically: Report of the International reflux study in children". Pediatrics, 139: 656, 2001.
14. ALADJEM, M.; BOICHIS, H.; HERTZ, H. y cols.: "The conservative management of vesicoureteric reflux: A review of 121 children". Pedaitrics, 65: 78, 1980.

15. KUCZYNSKA, R.; CZERWIONKA-SZAFLARSKA, M.: "Outcomes of conservative treatment of primary vesicoureteral reflux in children". Med. Sci. Monit., 6: 951, 2000.

16. ARANT, B.S.: "Medical management of mild and moderate vesicoureteral reflux: Follow up studies of infants and young children. A preliminary report of the southwest pediatric nephrology study group". J. Urol., 148: 1683, 1992.

17. LEVITT, S.B.; DUCKETT, J.; SPITZER, A. y cols.: "Medical versus surgical treatment of primary vesicoureteral reflux: A prospective international reflux study in children". J. Urol., 125: 277, 1981.

18. SJOSTROM, S.; SILLEN, U.; BACHELARD, M. y cols.: "Spontaneous resolution of high grade infantile vesicopureteral reflux". J. Urol., 172: 694, 2004.

19. MENON, P.; RAO, K.L.N.; BHATTACHARYA, A. y cols.: "Primary vesicoureteral reflux: Progress of disease, somatic growth and renal parameters". Indian Pedaitrics, 41:1025, 2004.

20. Mc LORIE, G.A.; Mc KENNA, P.H.; JUMPER, B.M. y cols.: "High grade vesicoureteral reflux: Analysis of observbational therapy". J. Urol., 144: 537, 1990.

21. GREENFIELD, S.P.; NG, M.; WANG, J.: "Resolution rates of low grade vesicoureteral reflux stratified by patient age at presentation". J. Urol., 157: 1410, 1997.

22. WENNERSTROM, M., HANSSON, S.; JODAL, U. y cols.: "Dissapearance of vesicoureteral reflux in children". Arch. Pediatr. Adolesc. Med., 152: 879, 1998. 